\title{
Biophysical Alterations in Lipid Rafts from Human Cerebral Cortex Associate with Increased BACE1/A $\beta P P$ Interaction in Early Stages of Alzheimer's Disease
}

\author{
Mario Díaz ${ }^{\mathrm{a}, *}$, Noemí Fabelo ${ }^{\mathrm{a}}$, Virginia Martín ${ }^{\mathrm{a}}$, Isidre Ferrer ${ }^{\mathrm{b}, \mathrm{c}}$, Tomás Gómez ${ }^{\mathrm{a}}$ and Raquel Marín ${ }^{\mathrm{d}}$ \\ ${ }^{a}$ Departamento de Biología Animal, Universidad de La Laguna, Tenerife, Spain \\ ${ }^{\mathrm{b}}$ Institut de Neuropatologia, IDIBELL-Hospital Universitari de Bellvitge, Universitat de Barcelona, \\ Hospitalet de Llobregat \\ ${ }^{\mathrm{c}}$ CIBERNED (Centro de Investigación Biomédica en Red de Enfermedades Neurodegenerativas), Spain \\ ${ }^{\mathrm{d}}$ Departamento de Fisiología, Universidad de La Laguna, Tenerife, Spain
}

Accepted 17 July 2014

\begin{abstract}
In the present study, we have assessed the biophysical properties of lipid rafts from different brain areas in subjects exhibiting early neuropathological stages of Alzheimer's disease (AD). By means of steady-state fluorescence polarization analyses using two environment-sensitive fluorescent probes, we demonstrate that lipid rafts from cerebellum, and frontal and entorhinal cortices, exhibit different biophysical behaviors depending on the stage of the disease. Thus, while membrane anisotropies were similar in the cerebellum along stages, lipid rafts from frontal and entorhinal cortices at AD stages I/II and AD III were significantly more liquid-ordered than in control subjects, both at the aqueous interface and hydrophobic core of the raft membrane. Thermotropic analyses demonstrated the presence of Arrhenius breakpoints between $28.3-32.0^{\circ} \mathrm{C}$, which were not influenced by the disease stage. However, analyses of membrane microviscosity $\left(\eta_{\text {app }}\right)$ demonstrate that frontal and entorhinal lipid rafts are notably more viscous and liquid-ordered all across the membrane from early stages of the disease. These physicochemical alterations in lipid rafts do not correlate with changes in cholesterol or sphingomyelin levels, but to reduced unsaturation index and increased saturate/polyunsaturated ratios in phospholipid acyl chains. Moreover, we demonstrate that $\beta$ secretase/A $\beta$ PP (amyloid- $\beta$ protein precursor) interaction and lipid raft microviscosity are strongly, and positively, correlated in $\mathrm{AD}$ frontal and entorhinal cortices. These observations strengthens the hypothesis that physical properties of these microdomains modulate the convergence of amyloidogenic machinery toward lipid rafts, and also points to a critical role of polyunsaturated fatty acids in amyloidogenic processing of A $\beta P P$.
\end{abstract}

Keywords: BACE1, $\beta$-secretase, cerebellum, docosahexaenoic acid, entorhinal cortex, fluorescence anisotropy, frontal cortex, lipid rafts, membrane viscosity, polyunsaturated fatty acids

\footnotetext{
${ }^{*}$ Correspondence to: Dr. Mario Diaz, U.D.I. Fisiología Animal, Departamento de Biología Animal, Universidad de La Laguna, 38206 Tenerife, Spain. Tel.: +34 922318343; Fax: +34 922318342; E-mail: madiaz@ull.es.
}

\section{INTRODUCTION}

Compelling evidence accumulated over the last two decades has pointed to lipid rafts, highly dynamic membrane assemblies enriched in cholesterol, and sphingolipids, as critical membrane domains involved in the regulation of $\mathrm{A} \beta \mathrm{PP}$ (amyloid- $\beta$ protein precursor) processing, and in the generation of the 
amyloid- $\beta$ (A $\beta$ ) peptide, considered the driving force in Alzheimer's disease (AD) pathology [1-5]. Although the majority of full-length A $\beta P P$ is compartmentalized to non-raft regions, an important subset of A $\beta P P$ partitions into lipid rafts along with BACE1 and $\gamma$-secretase components [4-6]. Integration of these membrane proteins into lipid rafts is determined by their capacity to establish specific protein-lipid interactions. Thus, both BACE1 and the $\gamma$-secretase subunits undergo posttranslational S-palmitoylation which aids their targeting to lipid raft domains [4, 6]. Furthermore, A $\beta P P$ contains a transmembrane domain resembling properties of canonical CRAC (Cholesterol Recognition/interaction Amino acid Consensus sequence) [7, 8], which allows the direct interaction with cholesterol - the major lipid component of lipid rafts [4, 7]. Additionally, Bhattacharyya et al. [9] have recently reported that a fraction of $\mathrm{A} \beta \mathrm{PP}$ undergoes post-translational palmitoylation and that this lipidmodified A $\beta P P$ is specifically enriched in lipid rafts.

The singular lipid structure of lipid rafts makes them liquid-ordered domains because the highly saturated phospholipid (including sphingomyelin) acyl chains and high cholesterol contents, enable closer lipid packing and rather restricted lateral movement, than in the surrounding non-raft regions [10]. Multiple lines of evidence, mostly based on selective lipid depletion in cultured cells, have suggested that these physicochemical features of lipid rafts provide the favorable environment for the amyloidogenic processing of $A \beta P P$ by altering the clustering dynamics of A $\beta P P-c l e a v i n g$ enzymes (reviewed in [1-5]). However, whether these alterations occur during the neuropathological progression leading to $\mathrm{AD}$ remain largely unknown.

Recently, we have demonstrated that lipid rafts from frontal cortex in late stages of Alzheimer's disease (Braak and Braak staging V/VI) display altered lipid profiles compared to control subjects [11]. Most lipid alterations were detected in the fatty acid composition of phospholipids, rather than in the raft lipid prototypical lipid classes cholesterol or sphingomyelin [11]. More recently, we have reported that alterations in lipid composition of lipid rafts from human cortex are detectable from very early stages of the disease, namely Braak and Braak stages I/II, where amyloid plaques and cognitive symptoms are still absent [12]. In these lipid rafts, we found complex lipid changes taking place in entorhinal and frontal cortices, but not in cerebellum, consistent with both, reduction in long chain polyunsaturated fatty acids (LCPUFA) in phospholipids, and moderate cholesterol depletion, which were accompanied by increased interaction of A $\beta P P$ and BACE [12]. These changes in the lipid matrix of lipid rafts are expected to impact their physicochemical properties and to alter membrane microdomain environment during $\mathrm{AD}$ progression, which might facilitate the co-segregation of $A \beta P P$ and BACE, eventually leading to increased $A \beta$ production.

Therefore, in the present study we have aimed to explore 1) the impact of lipid changes in lipid rafts during early stages of AD neuropathology, on the biophysical properties of lipid rafts, and 2) the possible relationships between biophysical changes and the convergence and association of proteins involved in the amyloidogenic processing of A $\beta P P$.

\section{MATERIALS AND METHODS}

\section{Human brain tissue and lipid rafts isolation}

Brain tissues were obtained from the Institute of Neuropathology Brain Bank (Bellvitge University Hospital, Spain) following the guidelines of the Hospital Ethics Committee. Based on neuropathological examination, 12 cases were classified into three categories according to Braak and Braak [13]: AD stages I/II (AD I group, average age $60.7 \pm 2.9$ years, of which one case was AD II), AD stage III (AD III group, average age $74.7 \pm 3.2$ years), and controls (CTRL group, average age $49.2 \pm 4.64$ years). A summary of all cases is shown in Table 1. With the sole exception of subject 12 , all cases were males. Regarding amyloid pathology, only one case (subject 7, staged I/A) displayed senile plaques in the neocortex (amyloid pathology stage A). Importantly, cases in this study were selected based on the results of our previous study aimed to determining the potential alterations of lipid composition of lipid rafts in early stages of AD [12]. Therefore, in doing this, it was possible to establish the precise biophysical correlates of lipid alterations. In every case, frontal cortex grey matter (area 8), entorhinal cortex, and cerebellar vermis were carefully dissected and separated from the subcortical white matter immediately at autopsy, and stored at $-80^{\circ} \mathrm{C}$ until use for lipid rafts isolation.

Lipid raft fractions were isolated following procedures adapted for human brain lipid rafts detailed in Martín et al. [11] and Ramírez et al. [14]. Fractions in the gradients were routinely tested for purity in western blot assays using different lipid raft and non-raft protein markers (i.e., anti-flotillin 1 and anti-caveolin- 1 for lipid rafts, anti- $\alpha 1 \mathrm{Na}+/ \mathrm{K}+$ ATPase subunit for nonraft plasma membrane, and anti-Hsp90 for cytosolic 
Table 1

Summary of cases included in the study

\begin{tabular}{lcccc}
\hline Case & Age & Gender & Postmortem delay & Neuropathology \\
\hline Control group & & & & \\
1 & 40 & Male & $5 \mathrm{~h} 10 \mathrm{~min}$ & $\mathrm{NL}$ \\
2 & 44 & Male & $6 \mathrm{~h} 40 \mathrm{~min}$ & $\mathrm{NL}$ \\
3 & 52 & Male & $3 \mathrm{~h}$ & $\mathrm{NL}$ \\
4 & 61 & Male & $3 \mathrm{~h} 50 \mathrm{~min}$ & $\mathrm{NL}$ \\
AD I/II group & & & & \\
5 & 67 & Male & $7 \mathrm{~h} 15 \mathrm{~min}$ & $\mathrm{II} / 0$ \\
6 & 61 & Male & $3 \mathrm{~h} 40 \mathrm{~min}$ & $\mathrm{I} / 0$ \\
7 & 53 & Male & $6 \mathrm{~h} 15 \mathrm{~min}$ & $\mathrm{I} / \mathrm{A}$ \\
8 & 62 & Male & $5 \mathrm{~h} 45 \mathrm{~min}$ & $\mathrm{I} / 0$ \\
AD III group & & & & \\
9 & 66 & Male & $2 \mathrm{~h} 45 \mathrm{~min}$ & $\mathrm{III} / 0$ \\
10 & 75 & Male & $5 \mathrm{~h} 30 \mathrm{~min}$ & $\mathrm{III} / 0$ \\
11 & 77 & Male & $6 \mathrm{~h} 20 \mathrm{~min}$ & $\mathrm{III} / 0$ \\
12 & 81 & Female & $1 \mathrm{~h} 30 \mathrm{~min}$ & III/0 \\
\hline
\end{tabular}

NL, no lesions. Neuropathology was classified according to Braak and Braak stages of Alzheimer's disease (stages I-III). 0-A indicates stages of amyloid neuropathology.

proteins). Fractions containing lipid raft markers flotillin-1 and caveolin-1 were collected, resuspended in isolation buffer and frozen at $-80^{\circ} \mathrm{C}$ until analyses.

\section{Lipid analyses}

Analyses of lipid composition of lipid rafts fractions was carried out as described previously by our group for human brain $[11,12]$. Total lipids were extracted with chloroform/methanol $(2: 1 \mathrm{v} / \mathrm{v})$ in the presence of $0.01 \%$ of butylated hydroxytoluene. Lipid classes were separated by one-dimensional double development high performance thin layer chromatography and quantified by densitometry. A fraction of total lipids extracted from lipid rafts were subjected to acidcatalyzed transmethylation to form the corresponding fatty acid methyl esters (FAME) and dimethyl acetals (DMA), which were purified by thin layer chromatography (TLC) and quantified by gas chromatograph using flame ionization detection and identified by mass spectrometry.

\section{Immunoprecipitation assays}

Lipid raft fractions were processed for immunoprecipitation as described in Fabelo et al. [12]. Briefly, fractions diluted in immunoprecipitation buffer $(50 \mathrm{mM}$ Tris- $\mathrm{HCl}$ pH $7.4 ; 150 \mathrm{mM} \mathrm{NaCl}$, $10 \%$ glycerol, $1 \%$ Nonidet-P 40, $1 \mathrm{mM}$ PMSF, and Roche's proteases inhibitor cocktail) were solubilized in $2 \%$ octyl $\beta$-D-glucopyranoside solution containing $\mathrm{Na}_{3} \mathrm{VO}_{4}$ and EDTA, and immunoprecipitated with an excess $(5 \mu \mathrm{g})$ of rabbit polyclonal anti-APP antibody
(Y-188, Abcam) overnight at $4^{\circ} \mathrm{C}$. Samples were then exposed to sheep anti-rabbit IgG dynabeads (Dynal). The resultant precipitated protein-dynabead immunocomplexes were disrupted using SDS loading buffer and resolved in $12.5 \%$ SDS-PAGE followed by western blotting. Proteins were then transferred to Hybond-P and blotted with rabbit anti-BACE polyclonal antibody (Chemicon International), and rabbit anti-Presenilin 1 antibody (Sigma-Aldrich), both diluted 1:500 in BLOTTO. Visualization of specific immunosignals developed for anti-rabbit horseradish peroxidaseconjugated secondary antibodies (diluted 1:10,000 in BLOTTO) were obtained with ECL chemiluminescence kit (Amersham, GE Healthcare). Densitometry values of specific signals were quantified using GS800 calibrated densitometer (Bio-Rad, Madrid, Spain). Relative values of BACE immunosignals were normalized to the total A $\beta P P$ content quantified in the same sample.

\section{Steady-state fluorescence anisotropy of lipid rafts}

Steady-state anisotropy $\left(r_{\mathrm{s}}\right)$ measurements were performed using two different fluorescent probes: nonpolar 1,6-diphenyl-1,3,5-hexatriene (DPH) and polar 1-[4-(trimethylammonium)phenyl]-6-phenyl1,3,5-hexatriene (TMA-DPH). DPH and TMA-DPH were dissolved in 1 tetrahydrofuran:1 ethanol (v/v) and stored as $200 \mu \mathrm{M}$ stock solutions. Fluorescent probes were used at $2 \mu \mathrm{M}$ in TBS $(20 \mathrm{mM}$ Tris- $\mathrm{HCl}$ buffer, pH 7.5, containing $150 \mathrm{mM} \mathrm{KCl)}$ prepared daily and stored protected from light until used. Lipid raft suspensions $(250 \mu \mathrm{L}, 50 \mu \mathrm{g}$ protein $/ \mathrm{mL})$ were incubated for $30 \mathrm{~min}$ at $37^{\circ} \mathrm{C}$ in PBS solutions labelled with the probes and under agitation. Fluorescence polarization values were determined using $355 \mathrm{~nm}$ excitation and $420 \mathrm{~nm}$ emission filters in an Appliskan multiplate reader (Thermo Scientific) equipped with polarizers. Unlabeled controls were simultaneously examined to correct for light scattering and intrinsic fluorescence. Steady-state fluorescence anisotropies were determined against temperature ramps from $20^{\circ} \mathrm{C}$ to $40^{\circ} \mathrm{C}$.

In some experiments, lipid rafts samples were preincubated with $5 \mathrm{mM}$ methyl- $\beta$-cyclodextrine (M $\beta C D$ ) in TBS at $37^{\circ} \mathrm{C}$ for $1 \mathrm{~h}$, before incorporation of the fluorescent probes.

\section{Microviscosity and thermodynamic calculations}

Membrane microviscosity, $\eta$, is the measure of frictional resistance to rotational and translational motions 
of molecules within bilayers. Membrane microviscosity (the reciprocal to membrane fluidity) can be estimated by fluorescence anisotropy of rod-shaped probes (as DPH or TMA-DPH), which reflects their rotational motion inside the membrane $[15,16]$. Because in lipid membranes rotational mobility of these probes is not isotropic but restricted in space, it also reflects the level of lipid order (or lipid packing) $[15,16]$. These two parameters, microviscosity and lipid order, discriminate different membrane phase states, such gel and liquid-ordered (which are believed to be responsible for functional lipid rafts domains), and fluid phase (in non-raft domains). In the present study, apparent microviscosity ( $\eta_{\text {app }}$ ) coefficients were computed based on the adaptation of the Perrin equation for rotational depolarization of DPH [17] and TMA-DPH [18] as described in Diaz et al. [19]. Discontinuity breakpoints $(T d)$, which inform on the temperature where phase transitions occur, and activation energies $(E a)$ for steady-state anisotropy were computed from Arrhenius plots [20], being the slopes of $\log \left(r_{\mathrm{s}}\right)$ or $\log \left(\eta_{\text {app }}\right)$ versus $1 / \mathrm{T}$ equal to $\mathrm{Ea} /(2.3 \mathrm{R})$. Flow activation energy or activation energy of viscous flow $(\mathrm{E} \eta)$ was derived by analogy to macroscopic fluids from empirical determination of temperature dependence of $\eta_{\text {app }}$, being the slope of $\log \left(\eta_{\text {app }}\right)$ versus $1 / \mathrm{T}$ plots equal to $\mathrm{E} \eta /(2.3 \mathrm{R})[16,21]$.

\section{Statistics}

Data were submitted to two-way ANOVA or oneway ANOVA followed by Student's $t$-test, where appropriate. Regression analyses were performed by non-linear regression. Pearson correlation coefficients $(R)$ and their corresponding $p$-values were calculated for all regression lines. Significance of regression analyses was assessed by one way ANOVA. When appropriate, $95 \%$ confidence intervals are indicated in Figs. 3 and 4.

\section{RESULTS}

\section{Thermotropic behavior of lipid rafts anisotropy}

We have used two different probes, the nonpolar probe DPH to determine the physical order at the hydrophobic core of the lipid raft membrane, and the polar TMA-DPH to assess rotational freedom at the membrane plane $[22,23]$. Results summarized in Fig. 1A show the temperature response of TMA-DPH steady-state anisotropy in lipid rafts from cerebellum (CB), entorhinal cortex (EC), and frontal cortex (FC) in the control, $\mathrm{AD}$ I/II, and AD III groups. Anisotropy curves in the range $20-40^{\circ} \mathrm{C}$ were fairly similar in the cerebellum irrespective of the neuropathological state, being the values measured at $37^{\circ} \mathrm{C}$ nearly identical in the three groups $\left(\mathrm{CB}, F_{2,5}=1.82, p>0.1\right)$. Conversely, in EC and FC, a clear upward shift in anisotropy curves was evident for controls as compared to AD groups, indicating that TMA-DPH anisotropies in lipid rafts from $\mathrm{AD}$ subjects are inherently higher in the whole range of temperatures than in control rafts (EC: $F_{2,5}=2.31, p=0.056 ; \mathrm{FC}: F_{2,5}=3.52$, $p<0.05)$. Arrhenius plots of steady-state TMA-DPH anisotropies revealed the existence of thermotropic phase transitions around discontinuity points $(T d)$, in the range $28.3-29.9^{\circ} \mathrm{C}$, which were quite similar between brain areas and also between disease stages (Fig. 1B). Similarly, computation of activation energies $(E a)$ below $T d$ indicated no differences between lipid rafts from either group.

Using a modified expression of Perrin equation for non-spherical probes $[18,21]$, we have assessed the changes in lipid raft microviscosity coefficients in the different brain areas and conditions (Table 1). Apparent microviscosity $\left(\eta_{\text {app }}\right)$ and flow activation energies $(\mathrm{E} \eta)$ in cerebellum were nearly constant $\left(\eta_{\text {app }}\right.$ around 1.71 poises at $37^{\circ} \mathrm{C}$ and $\mathrm{E} \eta$ around $2.5 \mathrm{kcal} / \mathrm{mol}$ ) and were unaffected by the disease (Fig. 1C). On the contrary, in EC and FC lipid rafts, $\eta_{\text {app }}$ values were significantly higher (average 1.82 poises at $37^{\circ} \mathrm{C}$ ) at $\mathrm{AD}$ stages $\mathrm{I} / \mathrm{II}$ and III/IV than in controls (Table 1), but these changes were not followed by changes in $\mathrm{E} \eta$, which remained in the range observed for cerebellum (Fig. 1C).

The thermotropic behavior of raft membranes at the internal core of the bilayers was assessed using DPH $[22,23]$. As in the case of TMA-DPH, lipid rafts displayed temperature-induced changes in membrane anisotropy, which were unaffected by the disease stage in $\mathrm{CB}\left(\mathrm{CB}, \mathrm{F}_{2,5}=0.84, p>0.1\right)$, but differed in EC and FC lipid rafts (EC: $F_{2,5}=5.53, p<0.05$; FC: $\left.\mathrm{F}_{2,5}=13.85, p<0.05\right)$. Thus, in cortical lipid rafts at stages I/II and III, DPH anisotropies were significantly higher in the whole temperature range as compared to controls (Fig. 2A). In order to get a deeper insight into the characterization of the mechanisms leading to these changes, we also explored the effects of cholesterol depletion in the same rafts using M $\beta C D$. The preincubation protocol with $\mathrm{M} \beta \mathrm{CD}$ used here ( $5 \mathrm{mM}$ for $1 \mathrm{~h}$ ), causes a $50 \%$ depletion of membrane cholesterol [24]. Treatment with M $\beta C D(+M \beta C D)$ brought about a considerable reduction of lipid raft probe anisotropies in all groups compared with controls (TBS), but the effects were more remarkable in 
A)

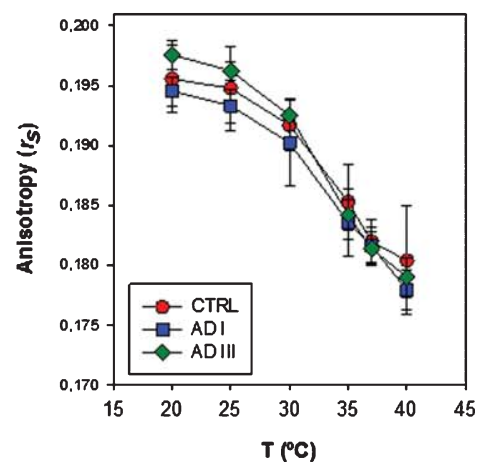

B)

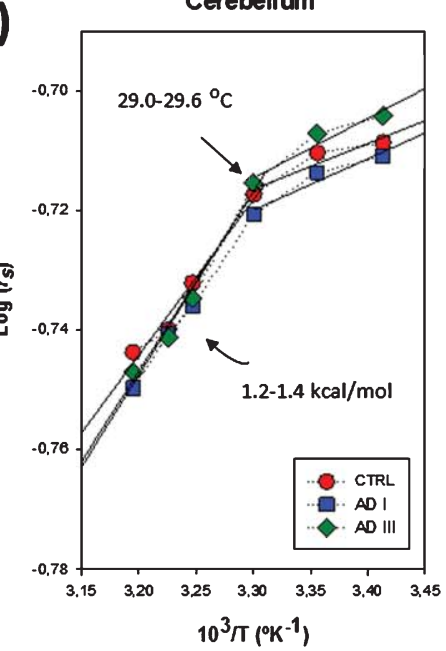

C)

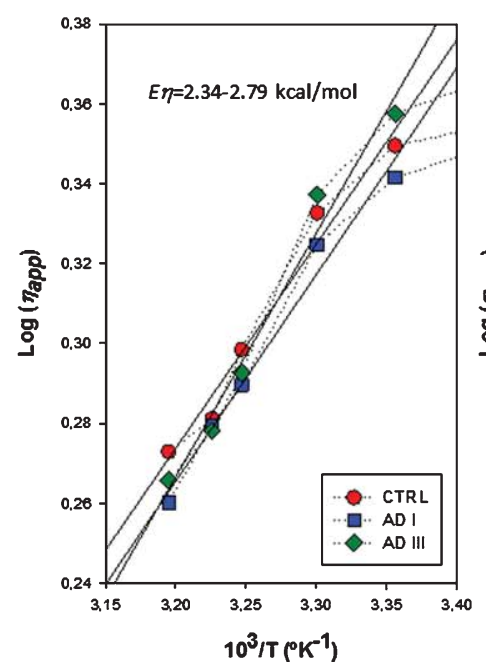

Entorhinal Cortex

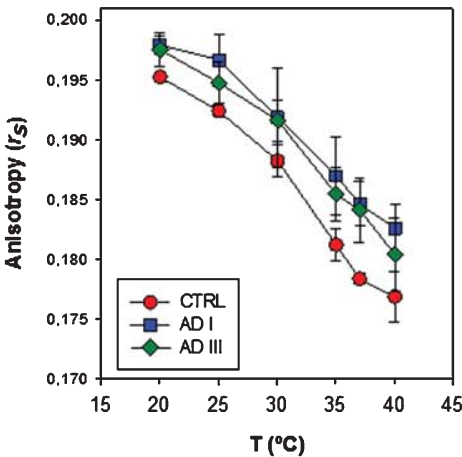

Entorhinal cortex

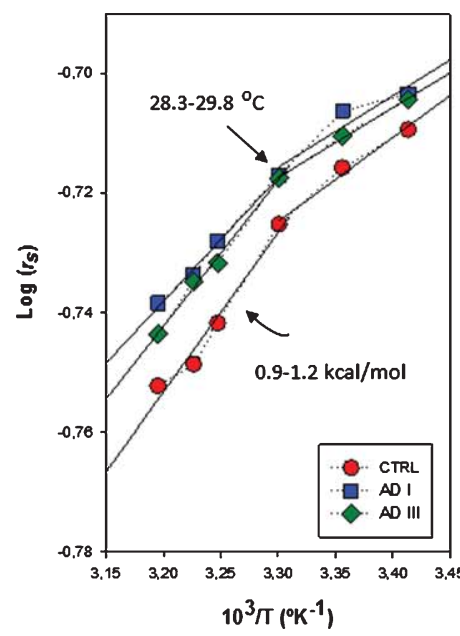

Entorhinal Cortex

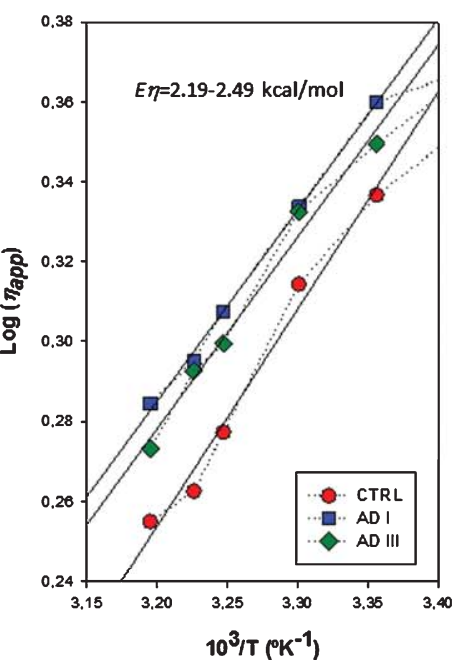

Frontal Cortex

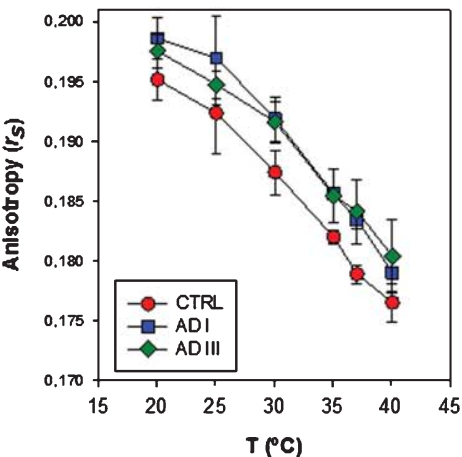

Frontal cortex

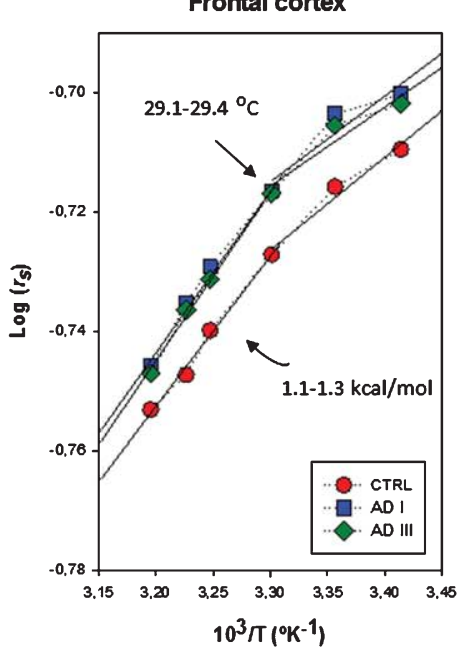

Frontal Cortex

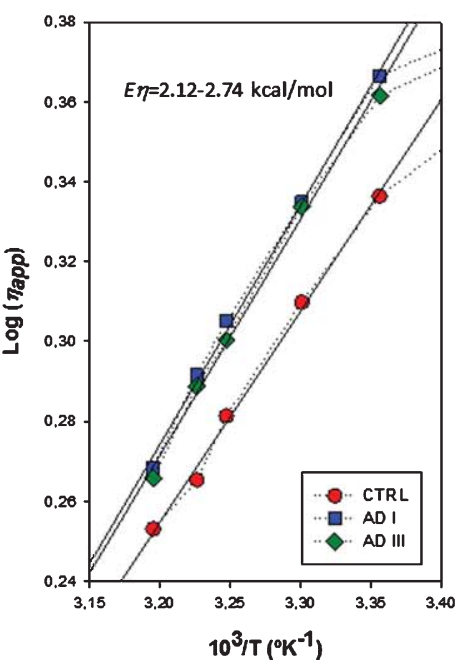

Fig. 1. Biophysical characterization of lipid rafts from cerebellum, entorhinal cortex, and frontal cortex in CTRL, AD I/II, and AD III brains probed with TMA-DPH. A) Temperature dependence of TMA-DPH fluorescence anisotropy of lipid rafts. B) Arrhenius plot for steady-state anisotropy in lipid rafts in the different brain areas. Arrows indicate discontinuity points $\left(T d\right.$ in $\left.{ }^{\circ} \mathrm{C}\right)$ and the range of activation energies $(E a$ in $\mathrm{kcal} / \mathrm{mol}$ ) obtained below $T d$. C) Apparent microviscosity $\left(\eta_{a p p}\right)$ analyses performed below $T d$ based on the Perrin equation modified for rod-shaped probes. Slopes indicate the activation energy of viscous flow $(E \eta)$. 
A)

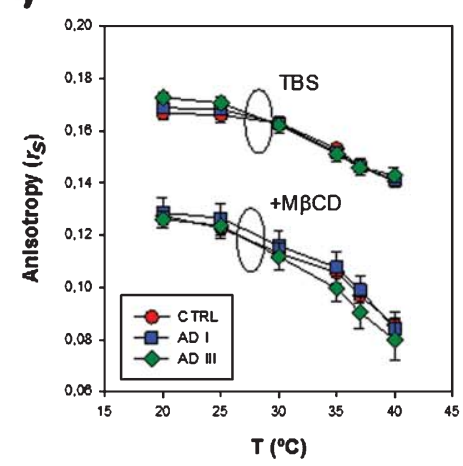

B)

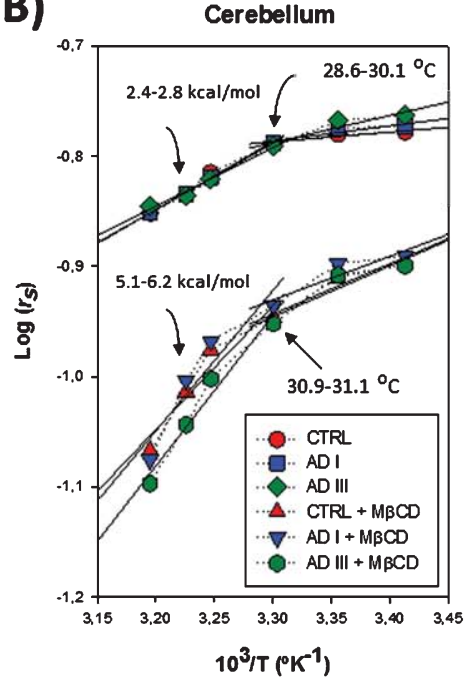

c)

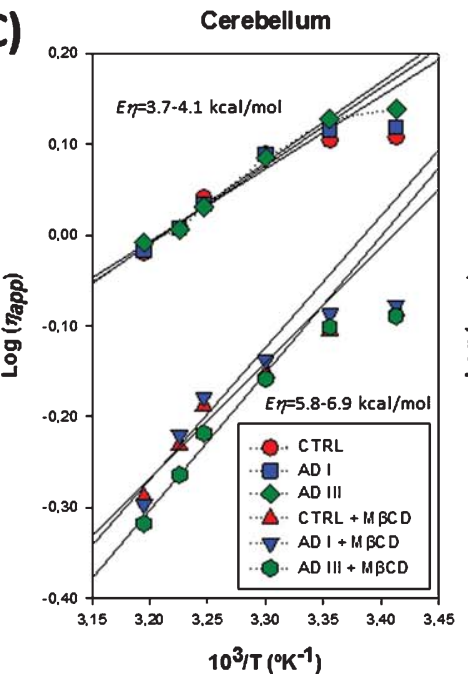

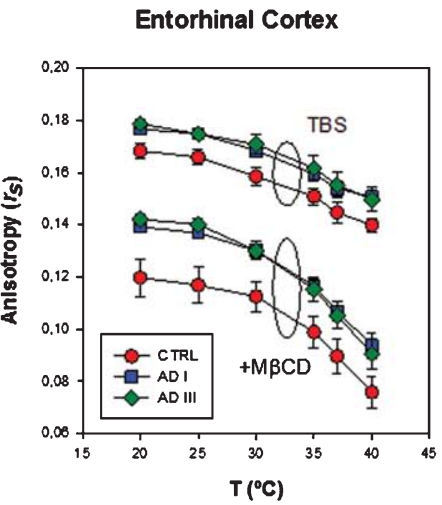
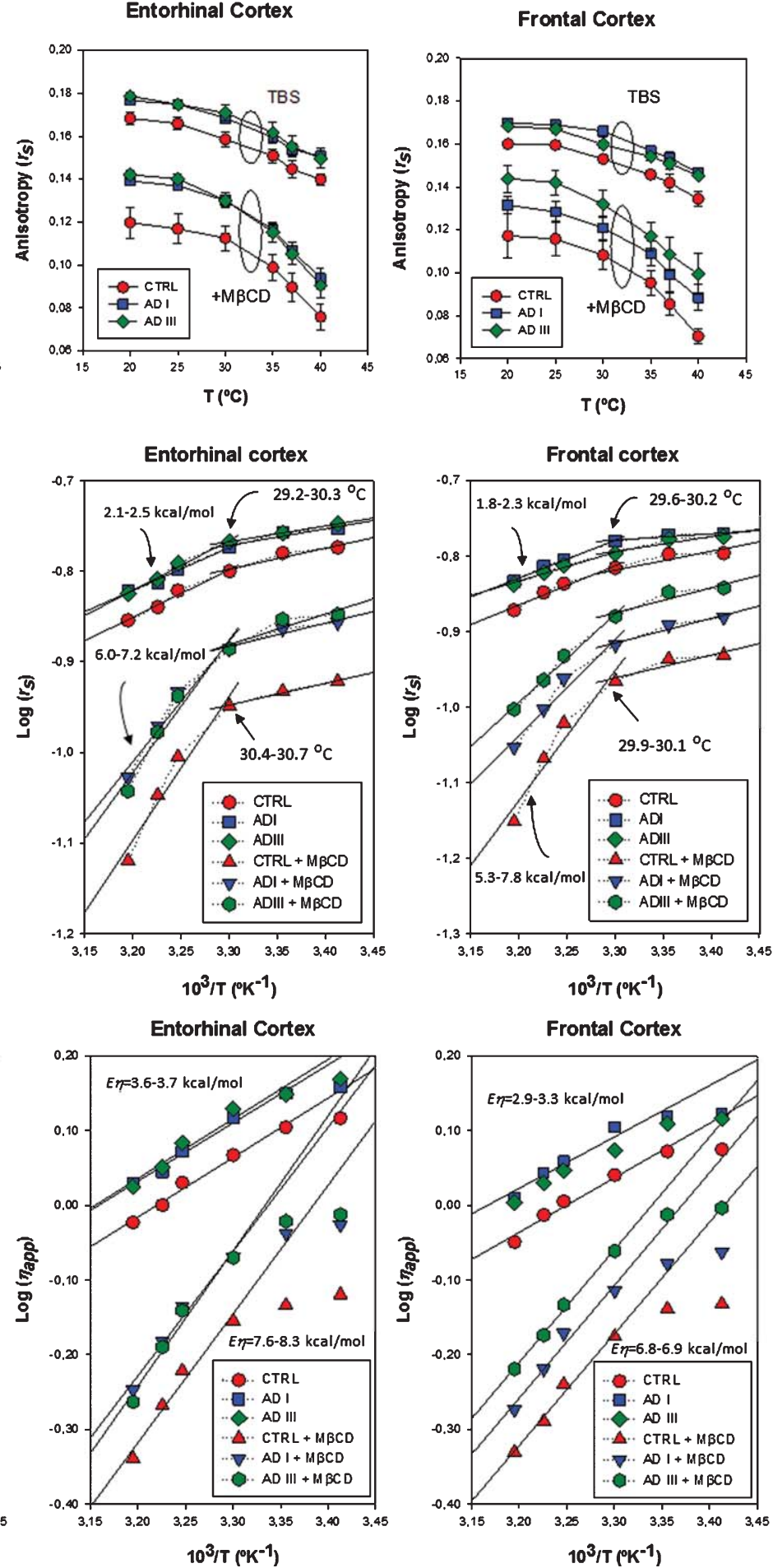

Fig. 2. Biophysical characterization of lipid rafts from cerebellum, entorhinal cortex, and frontal cortex in CTRL, AD I/II, and AD III brains probed with DPH. A) Temperature dependence of DPH fluorescence anisotropy of lipid rafts treated with methyl- $\beta$-cyclodextrin (M $\beta C D, 5$ $\mathrm{mM}$ ) or without (TBS, TRIS-buffered saline). B) Arrhenius plot for steady-state anisotropy in lipid rafts in the different brain areas and in the presence or absence of $\mathrm{M} \beta \mathrm{CD}$. Arrows indicate the position of discontinuity points $\left(T d\right.$ in $\left.{ }^{\circ} \mathrm{C}\right)$ and the range of activation energies $($ Ea in $\mathrm{kcal} / \mathrm{mol}$ ) obtained below $T d$. C) Apparent microviscosity $\left(\eta_{a p p}\right)$ analyses performed below $T d$. Slopes indicate the activation energy of viscous flow $(E \eta)$. 
$\mathrm{EC}$ and $\mathrm{FC}$ rafts from $\mathrm{AD}$ subjects $\left(\mathrm{CB}: F_{2,5}=1.89\right.$, $p>0.1 ; \mathrm{EC}: F_{2,5}=34.30, p<0.05 ; \mathrm{FC}: F_{2,5}=14.38$, $p<0.05$ ) (Fig. 2A). Arrhenius plots for steady-state DPH anisotropies also revealed the presence of discontinuity points in the range $29.2-30.7^{\circ} \mathrm{C}$ (Fig. 2B) which were shifted in all cases toward higher temperatures by $\mathrm{M} \beta \mathrm{CD}$ (range $31.5-32.7^{\circ} \mathrm{C}$ ). However, $\mathrm{M} \beta C D$ treatment caused a dramatic increase in activation energies $(E a)$ below $T d$, which increased by about $130 \%$ in CB lipid rafts. Moreover, In FC and EC lipid rafts, the reduction in $\log \left(r_{s}\right)$ and the increase in Ea induced by $\mathrm{M} \beta \mathrm{CD}$ were significantly higher in controls than in AD stages (Fig. 2B). Paralleling these changes in steady-state anisotropy, microviscosity values $\left(\eta_{\text {app }}\right)$ were dramatically reduced by M $\beta C D$ treatment in all cases, but the magnitude of changes differed between controls and AD lipid rafts (Table 1). Thus, in cerebellum, $\eta_{\text {app }}$ variations were very similar between stages (average 1.03 poises at $37^{\circ} \mathrm{C}$ in TBS and 0.58 poises at $37^{\circ} \mathrm{C}$ in $\mathrm{M} \beta C D$, representing a reduction of $43 \%$ or $\Delta \eta_{\text {app }}=-0.45$ poises), and these changes were equivalent in $\mathrm{FC}$ and $\mathrm{EC}$ lipid rafts from control brains (average 1.01 poises at $37^{\circ} \mathrm{C}$ in TBS versus 0.52 poises at $37^{\circ} \mathrm{C}$ in $\mathrm{M} \beta C D$, representing a $47 \%$ reduction and $\Delta \eta_{\text {app }}=-0.49$ poises at $37^{\circ} \mathrm{C}$ ). However, in AD lipid rafts from FC and EC, $\eta_{\text {app }}$ was significantly higher than in controls both under TBS (average $\Delta \eta_{\mathrm{app}}=+0.112$ poises at $37^{\circ} \mathrm{C}$ ) and $\mathrm{M} \beta \mathrm{CD}$ treatments (average $\Delta \eta_{\text {app }}=+0.117$ poises at $37^{\circ} \mathrm{C}$ ). Interestingly, $\Delta \eta_{\text {app }}$ values between TBS and $\mathrm{M} \beta C D$ in $\mathrm{AD}$ lipid rafts were in the range observed for control rafts $\left(\Delta \eta a p p=-0.47\right.$ poises at $\left.37^{\circ} \mathrm{C}\right)$. Further, pretreatment with $\mathrm{M} \beta \mathrm{CD}$ led to a considerable increase in $\mathrm{E} \eta$, which indicates that phase transitions must occur against a larger energy gradient (Fig. 2C). These differences are likely to reflect changes in the rotational order of DPH inside the membrane core, since DPH fluorescence and membrane depth location are largely independent of membrane cholesterol [23]. Overall, these results demonstrate that, under normal physiological conditions, lipid raft membrane viscosity and membrane dynamics are largely determined by its cholesterol content, and that lipid rafts from $\mathrm{EC}$ and $\mathrm{FC}$, but not from $\mathrm{CB}$, are intrinsically more viscous in $\mathrm{AD}$ brains from very early stages of the disease.

\section{Relationships between lipid raft lipids and microviscosity}

We next attempted to identify the biochemical correlates of these differential biophysical behaviors of lipid rafts, based on the alterations in their lipid profiles recently reported for these same subjects [12]. Using non-linear regression analysis, we assessed the linear relationships between relevant lipid components known to be altered in $\mathrm{AD}$ lipid rafts and $\eta_{\text {app }}$ variations (Fig. 3). First, using the whole dataset, we explored the relationship between $\eta_{\text {app }}$ determined at $37^{\circ} \mathrm{C}$, and cholesterol or sphingomyelin contents as independent variables (scatterplots in Fig. 3A, B). The results revealed positive correlations for both lipid constituents and $\eta_{\text {app }}$, which were statistically significant for cholesterol (TMA-DPH: $R=0.61, p<0.015$; DPH: $R=0.37, p<0.05$ ) (Fig. 3A). However, when lipid rafts data was structured according to disease stages, a negative correlation was observed for cholesterol (TMA-DPH: $R=-0.89, p<0.05$; DPH: $R=0.72$, $p<0.1$ ) and sphingomyelin (TMA-DPH: $R=-0.50$, $p>0.1$ ) (Fig. 3A, B, left plots), indicating that although lipid raft microviscosities increased in FC and EC from AD subjects, the alterations were not attributable to elevation of cholesterol or sphingomyelin levels. Indeed, we have observed that cholesterol contents in lipid rafts from $\mathrm{FC}$ and $\mathrm{EC}$ from $\mathrm{AD}$ subjects were decreased and, at least for FC, also those of sphingomyelin [12]. On the other hand, the phospholipid/cholesterol ratio (scatterplot in Fig. 3C) was also found to be positively related to $\eta_{\text {app }}$ (TMA-DPH: $R=0.30, p<0.05$; DPH: $R=0.36, p<0.05$ ), which apparently falls in contradiction with the observation that increased cholesterol content increases $\eta_{\text {app }}$. Given these anomalies, it would be expected that lipid rafts from $\mathrm{FC}$ and $\mathrm{EC}$ in $\mathrm{AD}$ were more fluid than in controls, which is obviously not the case.

The finding that phospholipid/cholesterol ratio was positively related to $\eta_{\mathrm{app}}$, pointed out to alterations in the degree of unsaturation of acyl chains in phospholipids being responsible for the changes in membrane microviscosity observed in lipid rafts from AD frontal and entorhinal cortices. To test this hypothesis, we first examined the relationship between unsaturation index (UI) and lipid rafts microviscosity. The results showed that reduction of UI is associated to significant increase in $\eta_{\text {app }}$, especially at the membrane aqueous interface (TMA-DPH: $R=0.62, p<0.005$; DPH: $R=0.45$, $p<0.01$ ) (Fig. 3D). As monoene fatty acids were unaffected by $\mathrm{AD}$ in any tissue or stage [12], the most likely factor accounting for the reduction in $\eta_{\text {app }}$ (and UI) were LCPUFA (long-chain polyunsaturated fatty acids). Accordingly, we observed a very significant reduction of $\eta_{\text {app }}$ as a function of n-3 LCPUFA (TMADPH: $R=0.55, p<0.005$; DPH: $R=0.54, p<0.005$ ) (Fig. 3E), and more consistently between $\eta_{\text {app }}$ and 

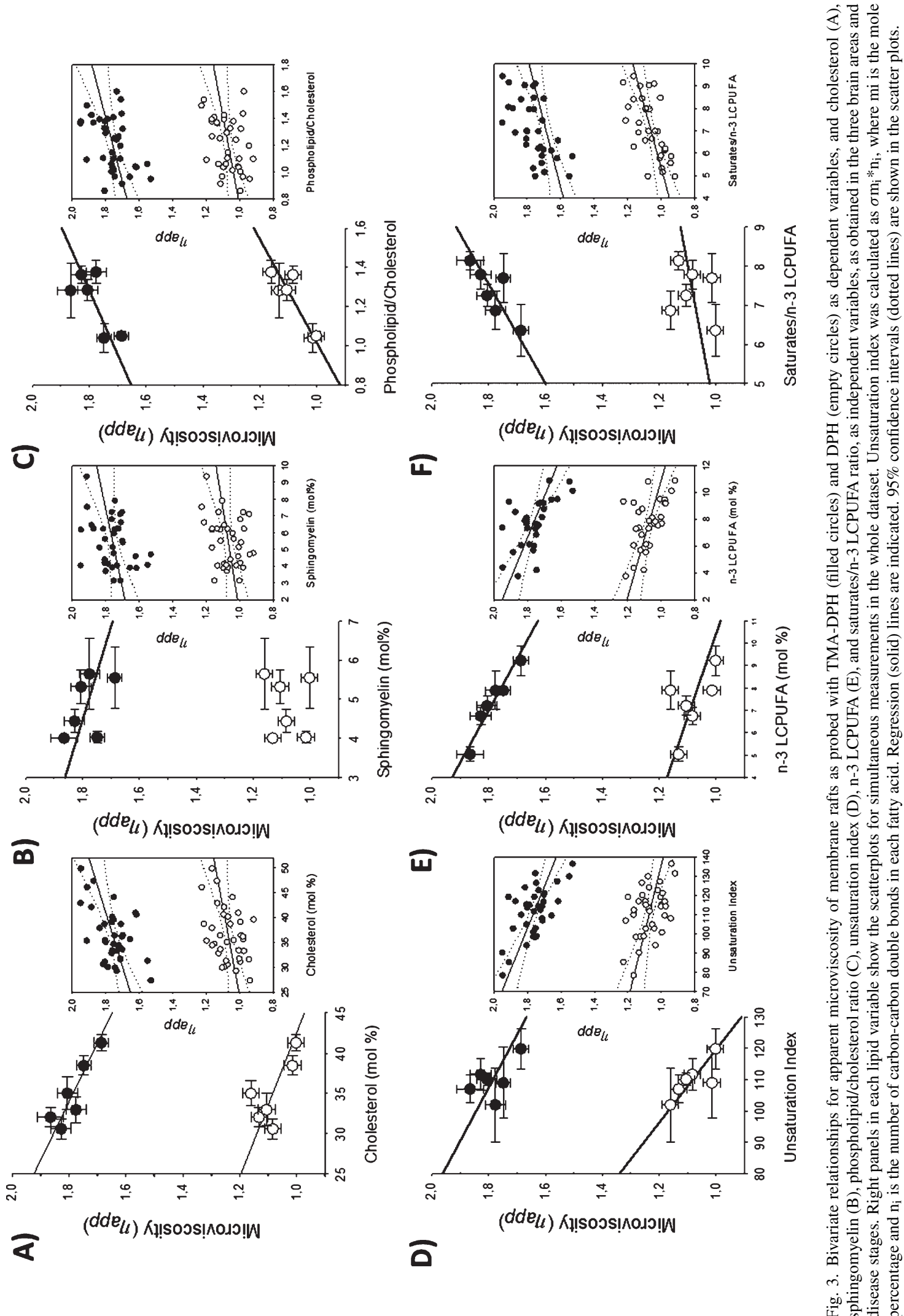
A)

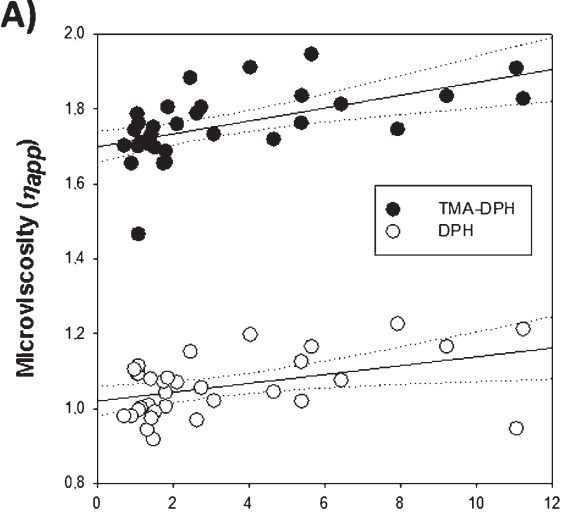

c)

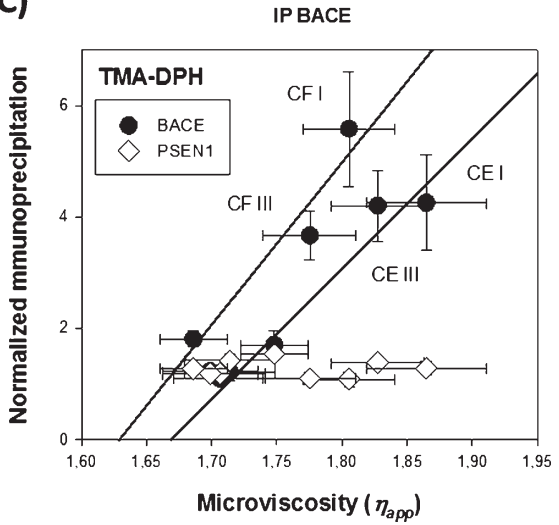

B)

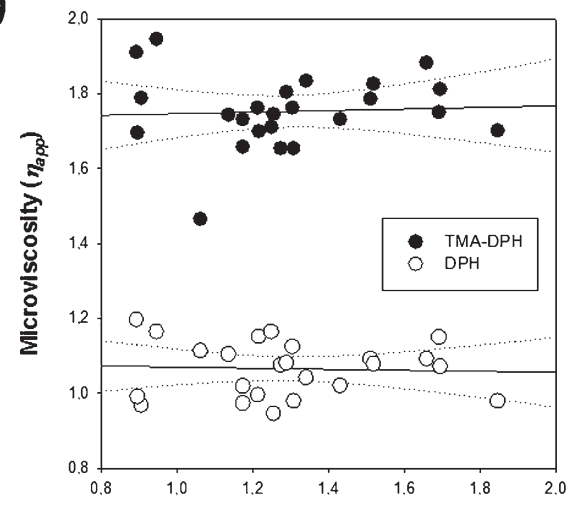

D)

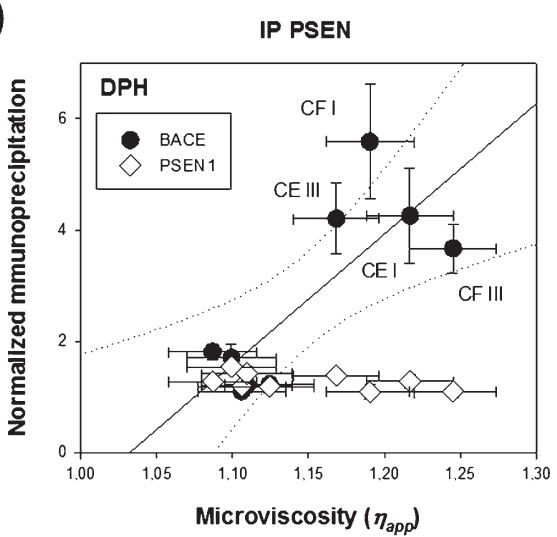

Fig. 4. Relationships between apparent microviscosity $\left(\eta_{a p p}\right)$ and amount of BACE1 and PSEN1 physically associated with A $\beta P P$, in lipid rafts. A, B) Scatterplots for simultaneous determinations of $\eta_{a p p}$, as determined at $37^{\circ} \mathrm{C}$ using TMA-DPH (solid circles) and DPH (empty circles), and BACE1 (A) and PSEN1 (B) immunoprecipated with AßPP, in the whole lipid rafts dataset. C, D) Regression analyses for the relationships between A $\beta$ PP-immunoprecipitated BACE1 (circles) and PSEN1 (diamonds), and lipid rafts microviscosity as probed with TMA-DPH (C) and DPH (D), in the different brain areas and disease stages. Regression (solid) lines are indicated. In C, the two regression lines correspond to frontal (CF, leftward line) and entorhinal cortex (EC, rightward line). 95\% confidence intervals (dotted lines) are shown in the scatterplots. 95\% Confidence intervals are indicated in $\mathrm{A}, \mathrm{B}$, and $\mathrm{D}$.

sat/n-3 LCPUFA (TMA-DPH: $R=0.62, p<0.001$; DPH: $R=0.64, p<0.001)($ Fig. $3 \mathrm{~F})$.

\section{Relationships between lipid raft microviscosity and $A \beta P P$ and $\beta / \gamma$-secretase clustering}

We finally assessed for the potential involvement of physicochemical alterations in lipid rafts on the degree of physical association of A $\beta P P$ and $\beta$ - and $\gamma$-secretases. First, by means of immunoprecipitation assays in the same lipid rafts, we observed that lipid rafts from entorhinal and frontal cortices at stages I/II and III, exhibit significantly increased degree of association between BACE1 and AßPP (Table 2). This augmented BACE1/A $\beta P P$ interaction occurred only in cortical lipid rafts, and was not detected in lipid rafts from cerebellum at any stage of the dis- ease (Table 2). Further, the interaction was specific for BACE1 and was not observed for PSEN1 beyond control values, which is in agreement with the alterations recently reported for these same subjects [12]. Moreover, BACE1/A $\beta P P$ interaction seemed to be influenced by the lipid environment of lipid rafts since a positive association was observed for the amount of immunoprecipitated BACE1 (but not PSEN1) and lipid raft microviscosity, especially at the aqueous interface (TMA-DPH: $R=0.62, p<0.001$ ) and hydrophobic core (DPH: $R=0.59, p<0.001$ ) of the membrane (Fig. 4A, B). These relationships were extended to the results obtained in lipid rafts from each brain area and disease stage. The outcomes revealed that lipid raft microviscosity was positively correlated to the degree of BACE1/A $\beta P P$ association in cortical areas, but not in cerebellum. Indeed, lipid rafts from FC 
Table 2

Apparent microviscosity values $\left(\eta_{a p p}\right)$ at $37^{\circ} \mathrm{C}$, calculated for the membrane plane and membrane hydrophobic core, as probed using TMA-DPH and DPH, respectively, in lipid rafts from controls and AD subjects at neuropathological stages I/II and III

\begin{tabular}{|c|c|c|c|c|}
\hline \multirow[t]{2}{*}{ Brain area } & \multirow[t]{2}{*}{ Stage } & \multirow[t]{2}{*}{ TMA-DPH } & \multicolumn{2}{|c|}{$\mathrm{DPH}$} \\
\hline & & & TBS & $\mathrm{M} \beta \mathrm{CD}$ \\
\hline \multirow[t]{3}{*}{ Cerebellum } & Ctrl & $1.706 \pm 0.035$ & $1.021 \pm 0.029$ & $0.588 \pm 0.021$ \\
\hline & AD I/II & $1.699 \pm 0.036$ & $1.040 \pm 0.029$ & $0.604 \pm 0.016$ \\
\hline & AD III & $1.714 \pm 0.034$ & $1.025 \pm 0.030$ & $0.546 \pm 0.010$ \\
\hline \multirow[t]{3}{*}{ Frontal cortex } & Ctrl & $1.748 \pm 0.025$ & $1.014 \pm 0.030$ & $0.541 \pm 0.015$ \\
\hline & AD I/II & $1.827 \pm 0.038^{*}$ & $1.083 \pm 0.028$ & $0.659 \pm 0.008^{*}$ \\
\hline & AD III & $1.865 \pm 0.046^{*}$ & $1.132 \pm 0.029^{*}$ & $0.647 \pm 0.009^{*}$ \\
\hline \multirow[t]{3}{*}{ Entorhinal cortex } & Ctrl & $1.686 \pm 0.026$ & $1.002 \pm 0.029$ & $0.515 \pm 0.012$ \\
\hline & AD I/II & $1.805 \pm 0.035^{*}$ & $1.105 \pm 0.020^{*}$ & $0.605 \pm 0.012 *$ \\
\hline & AD III & $1.775 \pm 0.034 *$ & $1.160 \pm 0.029^{*}$ & $0.671 \pm 0.007^{*}$ \\
\hline
\end{tabular}

Data were submitted to one way ANOVA followed by Student's $t$-test for comparisons with Ctrl group. * $p<0.05$ compared to Ctrl.

Table 3

Interactions of BACE1 and PSEN1 with A $\beta P P$ in lipid rafts obtained in brains from controls and $\mathrm{AD}$ subjects at neuropathological stages I/II and III

\begin{tabular}{lclc}
\hline Brain area & Stage & BACE1 & PSEN1 \\
\hline Cerebellum & Ctrl & $1.10 \pm 0.01$ & $1.19 \pm 0.04$ \\
& AD I/II & $1.23 \pm 0.08$ & $1.18 \pm 0.10$ \\
Frontal cortex & AD III & $1.21 \pm 0.15$ & $1.42 \pm 0.12$ \\
& Ctrl & $1.70 \pm 0.25$ & $1.54 \pm 0.04$ \\
Entorhinal cortex & AD I/II & $4.20 \pm 0.63^{* *}$ & $1.39 \pm 0.09$ \\
& AD III & $4.26 \pm 0.86^{* *}$ & $1.28 \pm 0.13$ \\
& AD I/II & $5.58 \pm 1.03^{* * *}$ & $1.09 \pm 0.13$ \\
& AD III & $3.67 \pm 0.44^{*}$ & $1.10 \pm 0.11$ \\
\hline
\end{tabular}

Data were submitted to ANOVA I followed by Student's $t$-test for comparisons with Ctrl group. * $p<0.05, * * p<0.01$, and $* * * p<0.005$ compared to Ctrl.

and EC at stages I/II and III, displaying highest levels of BACE1/A $\beta P P$ interaction, are closely related to largest apparent viscosity values, especially at the membrane plane (Fig. 4C). Paralleling these findings, A $\beta P P / P S E N$ association behaved rather independently of $\eta_{\mathrm{app}}$ in lipid raft (Fig. 4C, D).

\section{DISCUSSION}

In the present study, we provide new evidence that lipid rafts constitute subcellular targets seriously affected in AD neurodegeneration. We show that even at the earliest stages of $\mathrm{AD}$, physicochemical properties of raft membranes from entorhinal and frontal cortices exhibit severe alterations which might reflect initial events in the progression of the disease. It is widely accepted that lipid rafts are critical elements in the amyloidogenic processing of A $\beta P P$ [2, 4-6], and that specific lipid alterations within these domains accelerate $A \beta$ peptides generation thus facilitating aggregation $[1,3-5,25]$. However, the extents to which these lipid changes affect biophysical properties of lipid raft microenvironment and how this physicochemical scenario affect the dynamics of amyloid processing remain poorly understood.

By analyzing some of the biophysical properties of lipid rafts from control subjects, we show that lipid rafts from all brain areas display equivalent thermotropic behaviors, with phase transitions around a discontinuity point at $29^{\circ} \mathrm{C}$, and similar activation energies between brain areas as measured above $T d$. This homogeneity was observed both at the aqueous interface and hydrophobic core of the lipid rafts, being the viscosity of the internal core smaller and the flow activation energy larger than at the aqueous interface. These observations indicate a great degree of packing of membrane phospholipids, which may provide more abundant hydrophobic interactions between phospholipid fatty acids in the membrane plane, as it has been suggested in other membrane systems, including lipid rafts [19, 26, 27]. However, in AD brains, we have observed that lipid rafts membrane domains are differentially affected by the disease stage depending on the brain area considered. Thus, while cerebellar lipid rafts exhibit nearly constant biophysical and biochemical properties along AD neuropathological stages, rafts from enthorhinal and frontal cortices develop substantial changes in lipid composition that impact their biophysical properties, particularly their microviscosity. Strikingly, even at stages I/II, lipid rafts from frontal and entorhinal cortices appear as more viscous and liquid-ordered structures than in control counterparts. We also observed that these alterations project the whole transversal section of the lipid bilayer. From the neuropathological point of view, these findings are relevant since entorhinal cortex, which is one of the brain areas where neurofibrillary tangles first appear, are devoid of amyloid plaques at stages I/II [13, 28]. 
Therefore, biophysical changes must have occurred at initial stages of AD neuropathology, well before amyloid burden.

We have recently reported that lipid rafts from entorhinal and frontal cortices of stages I/II and III, contain significantly lower levels of n-3 and n6 LCPUFA (specifically docosahexaenoic acid and arachidonic acid, respectively), which, in turn, determine lower unsaturation and peroxidability indexes, and higher saturates/n-3 and saturates/n-6 indexes [12]. Importantly, most of these modifications observed at early stages are present, though to a different degree, in advanced stages (IV/V) of AD [11]. Noticeably, we have observed that lipid alterations also extend to lipid classes, since lipid rafts from AD I/II and AD III cortex display significantly lower cholesterol levels as well as increased phospholipid/cholesterol ratio, as compared to controls. The reasons for these wide lipid alterations are currently unknown, but they suggest that disruption of neuronal lipid homeostasis $[2,3$, 29-31], concomitantly with oxidative damage of membrane lipids [32-35], convey to overwhelm neuronal capacity to adapt membrane biosynthetic/recycling mechanisms needed to maintain lipid raft homeostasis.

It can be surmised that these biochemical changes are likely to severely affect physicochemical microenvironment of lipid rafts. One strength in the present study was that we have performed measurements of biophysical features within the same lipid rafts where we have previously identify lipid alterations associated with early stages of AD [12], which allowed assessment on the extent to which changes in each lipid variable impacted membrane order. Thus, by analyzing lipid raft data as a whole, we demonstrate that increasing cholesterol or sphingomyelin contents in lipid rafts causes the expected increase in membrane viscosity [36-38], and that augmented levels of polyunsaturated fatty acids or elevation of unsaturation index brings about more fluid raft membranes [19, 39, 40]. Taken together, our data indicate that the increased microviscosity observed in lipid rafts from entorhinal and frontal cortices in AD stages I/II and III, cannot be attributed to changes in cholesterol or sphingomyelin levels (which in fact were reduced in these rafts). In agreement, we show that forced depletion of cholesterol using $\mathrm{M} \beta \mathrm{CD}$, provoked a considerable reduction of microviscosity in lipid rafts from all origins, and this effect was accompanied by a two-fold increase in E $\eta$, which highlights the central role of cholesterol in setting the thermodynamic properties of liquid-crystal phase characteristic of ordered lipid rafts [37, 41]. Therefore, with cholesterol being reduced in untreated
FC and EC lipid rafts, but activation energies $(E a)$ and flow activation energies $(\mathrm{E} \eta)$ being similar to control rafts, some sort of alternative mechanisms must have been occurred to augment the microviscosity state of these lipid rafts, likely involving other components in the membrane lipid matrix. We found the explanation on the reduction in the degree of unsaturation of lipid raft phospholipids, specifically for n-3 LCPUFA, and the proportional increase in the saturates/n-3 LCPUFA. In agreement, best bivariate relationships for $\eta_{\text {app }}$ as dependent variable were observed for n-3 LCPUFA and saturates/n-3 LCPUFA relationship, especially at the membrane plane. Interestingly, we have recently demonstrated a very similar physicochemical behavior in lipid rafts from frontal cortex of aged A $\beta P P / P S 1$ transgenic mice, a familial model of AD [19, 42]. Therefore we conclude that lipid rafts from FC and $\mathrm{EC}$ in $\mathrm{AD}$ brains are intrinsically more viscous and ordered than in control subjects due to the depletion in polyunsaturated fatty acids and the secondary increase in saturates/n-3 LCPUFA ratio.

The pathological impact of the physiochemical alterations discussed above was demonstrated by the study of the dynamics of associations of proteins directly involved in amyloid production in the same lipid rafts. We had previously observed that BACE1 and PSEN interact with $\mathrm{A} \beta P P$ at the raft membranes, even in control brains, but noticeably, that the degree of physical association between A $\beta P P$ and BACE1 was positively modulated in FC and EC of AD brains, even at the earliest stages of the disease [12]. Here, we found that such interaction occurs in consonance with changes in the lipid raft microenvironment. Thus, simultaneous measurements of BACE1 or PSEN1 immunoprecipitated with A $\beta P P$ and apparent microviscosity reveals that the amount of BACE1 present in lipid rafts increases linearly with $\eta_{\mathrm{app}}$, while the amount of PSEN1 in lipid rafts remained unaltered. A $\beta P P / B A C E 1$ association was better correlated with the viscosity at the aqueous interface than at hydrophobic core of the bilayer, suggesting interactions of their respective transmembrane segments with long chain saturated fatty acids, which in turn are facilitated for interaction upon reduction of membrane phospholipids polyunsaturation. Obviously, factors promoting physical contact between A $\beta P P$ and $\beta$-secretase within rafts are expected to enhance CTF $\beta 99$ intermediate formation and to increase substrate availability for further cleavage by $\gamma$-secretase [5, 41, 43]. Therefore, we postulate that it is the viscous lipid rafts environment, rather than their lipid components themselves, what determines the ease of amyloidogenic 
processing of A $\mathrm{APP}$ within rafts. In line with this hypothesis, our present data are also reconciling in relation to conflicting evidence describing opposite effects of cholesterol on the regulation of A $\beta P P$ processing $[1,3,25]$. Thus, it has been solidly demonstrated that cholesterol enrichment increases membrane viscosity $[1,37,41]$, and this can explain the displacement of $\mathrm{A} \beta \mathrm{PP}$ processing toward the amyloidogenic cleavage and $\mathrm{A} \beta$ formation $[5,44,45]$. Conversely, work from other laboratories have shown that neuronal membrane cholesterol loss enhances amyloid peptide generation $[25,46]$, and this, as we show here, may also be associated to reduced membrane fluidity as long as membrane unsaturation is concurrently decreased, thus paving a favorable environment for amyloidogenic A $\beta P P c$ cleavage. Nonetheless, as we demonstrate in the present study, the role of cholesterol in lipid rafts extends well beyond changes of membrane fluidity, being also linked to thermodynamic behavior of these microdomains, including setting of flow activation energies, determination of gel/liquid-crystalline phase transition, and stabilization of sphingomyelinrich lipid matrix in the raft membranes.

The causes for the changes in the physicochemical properties of lipid rafts in human brain cortex at initial stages of $\mathrm{AD}$ neurodegeneration are currently unknown. Certainly, membrane microviscosity is a homeostatic parameter resulting from complex lipid interactions within the bilayer under the influence of physical parameters. The intricate lipid rafts nanoscopic dynamics, suggest that lipid alterations may well occur at different subcellular stages in the biosynthetic pathway, from vesicle trafficking/sorting and phase segregation at the trans Golgi network, to phospholipid remodeling as consequence of acylation/deacylation mechanisms [47-49]. However, given that one major determinant in the biophysical alterations observed here is the depletion in phospholipid polyunsaturation, it is tempting to speculate that either (lipo)peroxidative mechanisms alter the saturated/unsaturated equilibrium at the membrane or that the incorporation of LCPUFA into phospholipids is reduced at these initial stages of the disease. In any case, it is likely that the extremely low capacity of human brain to synthesize LCPUFA [50], especially those of the $n-3$ series, critically limits the ability of nerve cells to correct such membrane defects.

In summary, our present study demonstrates that A $\beta P P / B A C E 1$ association is significantly favored, if not promoted, by the physical microenvironment of lipid rafts. The finding that lipid rafts microviscosity is positively related to the degree of physical association of $A \beta P P$ and BACE1 in lipid rafts where cholesterol levels are reduced, demonstrates essential role of $n-3$ LCPUFA in the biophysical properties of lipid rafts in neuronal physiology. Understanding the mechanisms leading to changes in lipid raft membrane's biophysics and how they affect A $\beta P P$ processing, should provide new clues for early detection and insights to tailoring new therapeutic strategies for prevention and treatment of $\mathrm{AD}$.

\section{ACKNOWLEDGMENTS}

This study was supported by research grants SAF2010-22114-C02-01/02 from Ministerio de Economía y Competitividad (MINECO, SPAIN) and by the Seventh Framework Programme of the European Commission (grant agreement 278486: DEVELAGE). We thank Dr. Fernando Lahoz (Departmento de Física Fundamental y Experimental, Universidad de La Laguna) for helpful comments and discussion on the anisotropy analyses. We are grateful to CEI-Canarias, Campus Atlántico Tricontinental (Universidad de La Laguna) and to Biosearch Life-Puleva Biotech for continuous support to the project at the Laboratory of Membrane Physiology and Biophysics.

Authors' disclosures available online (http://www.jalz.com/disclosures/view.php?id=2456).

\section{REFERENCES}

[1] Wood WG, Schroeder F, Igbavboa U, Avdulov NA, Chochina SV (2002) Brain membrane cholesterol domains, aging and amyloid beta-peptides. Neurobiol Aging 23, 685694.

[2] Cordy JM, Hooper NM, Turner AJ (2006) The involvement of lipid rafts in Alzheimer's disease. Mol Membr Biol 23, 111-122.

[3] Di Paolo G, Kim TW (2011) Linking lipids to Alzheimer's disease: Cholesterol and beyond. Nat Rev Neurosci 12, 284296.

[4] Rushworth JV, Hooper NM (2011) Lipid rafts: Linking Alzheimer's amyloid- $\beta$ production, aggregation, and toxicity at neuronal membranes. Int J Alzheimers Dis 2011, 603052 .

[5] Hicks DA, Nalivaeva NN, Turner AJ (2012) Lipid rafts and Alzheimer's disease: Protein-lipid interactions and perturbation of signaling. Front Physiol 3, 189.

[6] Vetrivel KS, Thinakaran G (2010) Membrane rafts in Alzheimer's disease beta-amyloid production. Biochim Biophys Acta 1801, 860-867.

[7] Beel AJ, Sakakura M, Barrett PJ, Sanders CR (2010) Direct binding of cholesterol to the amyloid precursor protein: An important interaction in lipid-Alzheimer's disease relationships? Biochim Biophys Acta 1801, 975-982.

[8] Fantini J, Barrantes FJ (2013) How cholesterol interacts with membrane proteins: An exploration of cholesterol-binding 
sites including CRAC, CARC, and tilted domains. Front Physiol 4,31 .

[9] Bhattacharyya R, Barren C, Kovacs DM (2013) Palmitoylation of amyloid precursor protein regulates amyloidogenic processing in lipid rafts. J Neurosci 33, 11169-11183.

[10] Pike LJ (2006) Rafts defined: A report on the Keystone Symposium on Lipid Rafts and Cell Function. J Lipid Res 47, 1597-1598.

[11] Martín V, Fabelo N, Santpere G, Puig B, Marín R, Ferrer I, Díaz M (2010) Lipid alterations in lipid rafts from Alzheimer's disease human brain cortex. $J$ Alzheimers Dis 19, 489-502.

[12] Fabelo N, Martín V, Marín R, Moreno D, Ferrer I, Díaz M (2014) Altered lipid composition in cortical lipid rafts occurs at early stages of sporadic Alzheimer's disease and facilitates APP/BACE1 interactions. Neurobiol Aging 35, 1801-1812.

[13] Braak H, Braak E (1991) Neuropathological stageing of Alzheimer-related changes. Acta Neuropathol 82, 239-259.

[14] Ramírez CM, González M, Díaz M, Alonso R, Ferrer I, Santpere G, Puig B, Meyer G, Marin R (2009) VDAC and $\mathrm{ER} \alpha$ interaction in caveolae from human cortex is altered in Alzheimer's disease. Mol Cell Neurosci 42, 172-183.

[15] Demchenko AP, Mély Y, Duportail G, Klymchenko AS (2009) Monitoring biophysical properties of lipid membranes by environment-sensitive fluorescent probes. Biophys J 96, 34613470.

[16] Szöllosi J (1994) Fluidity/viscosity of biological membranes. In Mobility and Proximity in Biological Membranes, Damjanovich S, Edidin M, Szollosi J, Tron L, eds. CRC Press, Boca Ratón, FL, pp. 137-208.

[17] Laat SW, Saag PT, Shinitzky M (1977) Microviscosity modulation during the cell cycle of neuroblastoma cells. Proc Natl Acad Sci U S A 10, 4458-4461.

[18] Chazotte B (1994) Comparisons of the relative effects of polyhydroxyl compounds on local versus long-range motions in the mitochondrial inner membrane. Fluorescence recovery after photobleaching, fluorescence lifetime and fluorescence anisotropy studies. Biochim Biophys Acta 1194, 315-328.

[19] Diaz ML, Fabelo N, Marín R (2012) Genotype-induced changes in biophysical properties of frontal cortex lipid raft from APP/PS1 transgenic mice. Front Physiol 3, 454.

[20] Almansa E, Sánchez JJ, Cozzi S, Rodríguez C, Díaz M (2003) Temperature activity relationship for the intestinal $\mathrm{Na}+\mathrm{K}+$-ATPase of Sparus aurata. A role for the phospholipid microenvironment? J Comp Physiol B 173, 231-237.

[21] Shinitzky M, Barenholz Y (1974) Dynamics of the hydrocarbon layer in liposomes of lecithin and sphingomyelin containing dicetylphosphate. J Biol Chem 249, 2652-2657.

[22] Lentz BR (1993) Use of fluorescent probes to monitor molecular order and motions within liposome bilayers. Chem Phys Lipids 64, 99-116.

[23] Kaiser RD, London E (1998) Location of diphenylhexatriene (DPH) and its derivatives within membranes: Comparison of different fluorescence quenching analyses of membrane depth. Biochemistry 37, 8180-8190.

[24] Zidovetzki R, Levitan I (2007) Use of cyclodextrins to manipulate plasma membrane cholesterol content: Evidence, misconceptions and control strategies. Biochim Biophys Acta 1768, 1311-1324.

[25] Abad-Rodriguez J, Ledesma MD, Craessaerts K, Perga S, Medina M, Delacourte A, Dingwall C, De Strooper B, Dotti CG (2004) Neuronal membrane cholesterol loss enhances amyloid peptide generation. J Cell Biol 167, 953-960.

[26] Sonnino S, Prinetti A (2010) Lipids and membrane lateral organization. Front Physiol 1, 153.
[27] Van Blitterswijk WJ, van der Meer BW, Hilkmann H (1987) Quantitative contributions of cholesterol and the individual classes of phospholipids and their degree of fatty acyl (un)saturation to membrane fluidity measured by fluorescence polarization. Biochemistry 26, 1746-1756.

[28] Serrano-Pozo A, Frosch MP, Masliah E, Hyman BT (2011) Neuropathological alterations in Alzheimer disease. Cold Spring Harb Perspect Med 1, a006189.

[29] Bennett SA, Valenzuela N, Xu H, Franko B, Fai S, Figeys D (2013) Using neurolipidomics to identify phospholipid mediators of synaptic (dys)function in Alzheimer's Disease. Front Physiol 4, 168.

[30] Corrigan FM, Horrobin DF, Skinner ER, Besson JA, Cooper MB (1998) Abnormal content of $\mathrm{n}-6$ and $\mathrm{n}-3$ long-chain unsaturated fatty acids in the phosphoglycerides and cholesterol esters of parahippocampal cortex from Alzheimer's disease patients and its relationship to acetyl CoA content. Int $J$ Biochem Cell Biol 30, 197-207.

[31] Piccinini M, Scandroglio F, Prioni S, Buccinná B, Loberto N, Aureli M, Chigorno V, Lupino E, DeMarco G, Lomartire A, Rinaudo MT, Sonnino S, Prinetti A (2010) Deregulated sphingolipid metabolism and membrane organization in neurodegenerative disorders. Mol Neurobiol 41, 314-340.

[32] Zampagni M, Evangelisti E, Cascella R, Liguri G, Becatti M, Pensalfini A, Uberti D, Cenini G, Memo M, Bagnoli S, Nacmias B, Sorbi S, Cecchi C (2010) Lipid rafts are primary mediators of amyloid oxidative attack on plasma membrane. J Mol Med 88, 597-608.

[33] Ferrer I (2009) Altered mitochondria, energy metabolism, voltage-dependent anion channel, and lipid rafts converge to exhaust neurons in Alzheimer's disease. J Bioenerg Biomembr 41, 425-431.

[34] Sultana R, Perluigi M, Allan Butterfield D (2013) Lipid peroxidation triggers neurodegeneration: A redox proteomics view into the Alzheimer disease brain. Free Radic Biol Med 62, 157-169.

[35] Aso E, Lomoio S, López-Gonzalez I, Joda L, Carmona M, Fernández-Yagüe N, Moreno J, Juvés S, Pujol A, Pamplona R, Portero-Otín M, Martín V, Diaz M, Ferrer I (2012) Amyloid generation and dysfunctional immunoproteasome activation with disease progression in animal model of familial Alzheimer's disease. Brain Pathol 22, 636-653.

[36] Barenholz Y, Thompson TE (1980) Sphingomyelins in bilayers and biological membranes. Biochim Biophys Acta 604, 129-158.

[37] Ohvo-Rekilä H, Ramstedt B, Leppimäki P, Slotte JP (2002) Cholesterol interactions with phospholipids in membranes. Prog Lipid Res 41, 66-97.

[38] Finegold L (1993) Cholesterol in Membrane Models. CRC Press, Boca Raton, FL, USA.

[39] Shaikh SR (2012) Biophysical and biochemical mechanisms by which dietary $\mathrm{N}-3$ polyunsaturated fatty acids from fish oil disrupt membrane lipid rafts. J Nutr Biochem 23, 101-105.

[40] Corsetto PA, Cremona A, Montorfano G, Jovenitti IE, Orsini F, Arosio P, Rizzo AM (2012) Chemical-physical changes in cell membrane microdomains of breast cancer cells after omega-3 PUFA incorporation. Cell Biochem Biophys 64, 4559.

[41] Askarova S, Yang X, Lee JC (2011) Impacts of membrane biophysics in Alzheimer's disease: From amyloid precursor protein processing to $A \beta$ peptide-induced membrane changes. Int J Alzheimers Dis 2011, 134971

[42] Fabelo N, Martín V, Marín R, Santpere G, Aso E, Ferrer I, Díaz M (2012) Evidence for premature "Lipid raft aging" in APP/PS1 double transgenic mice, a familial model 
of Alzheimer's disease. J Neuropathol Exp Neurol 71, 868-881.

[43] von Arnim CA, von Einem B, Weber P, Wagner M, Schwanzar D, Spoelgen R, Strauss WL, Schneckenburger H (2008) Impact of cholesterol level upon APP and BACE proximity and APP cleavage. Biochem Biophys Res Commun 370, 207-212.

[44] Simons M, Keller P, De Strooper B, Beyreuther K, Dotti CG, Simons K (1998) Cholesterol depletion inhibits the generation of beta-amyloid in hippocampal neurons. Proc Natl Acad Sci U S A 95, 6460-6464.

[45] Ehehalt R1, Keller P, Haass C, Thiele C, Simons K (2003) Amyloidogenic processing of the Alzheimer beta-amyloid precursor proteindepends on lipid rafts. J Cell Biol 160, 113123.

[46] Ledesma MD, Dotti CG (2005) The conflicting role of brain cholesterol in Alzheimer's disease: Lessons from the brain plasminogen system. Biochem Soc Symp 72, 129-138.
[47] Diaz-Rohrer BB, Levental KR, Simons K, Levental I (2014) Membrane raft association is a determinant of plasma membrane localization. Proc Natl Acad Sci U S A 111, 8500-8505.

[48] Sonnino S, Prinetti A (2013) Membrane domains and the "lipid raft" concept. Curr Med Chem 20, 4-21.

[49] Kitson AP, Stark KD, Duncan RE (2012) Enzymes in brain phospholipid docosahexaenoic acid accretion: A PL-ethora of potential PL-ayers. Prostaglandins Leukot Essent Fatty Acids 87, 1-10.

[50] Alessandri JM, Guesnet P, Vancassel S, Astorg P, Denis I, Langelier B, Aïd S, Poumès-Ballihaut $C$, Champeil-Potokar G, Lavialle M (2004) Polyunsaturated fatty acids in the central nervous system: Evolution of concepts and nutritional implications throughout life. Reprod Nutr Dev 44, 509-538. 